\title{
HIF-1 a Promotes the Metastasis of Esophageal Squamous Cell Carcinoma by Targeting SP1
}

\author{
Xueting $\mathrm{Hu}^{1,2^{*}}$, Jiatong $\operatorname{Lin}^{1,2^{*}}$, Ming Jiang ${ }^{3^{*}}$, Xiaotian $\mathrm{He}^{1,2}$, Kefeng Wang ${ }^{1,2}$, Wenjian Wang1,2, Chuwen \\ $\mathrm{Hu}^{1,2}$, Zhiwen Shen ${ }^{1,2}$, Zhanghai He ${ }^{1,2}$, Huayue $\operatorname{Lin}^{1,4}$, Duoguang $\mathrm{Wu}^{1,2}$, Minghui Wang ${ }^{1,2}{ }^{\varpi}$
}

1. Guangdong Provincial Key Laboratory of Malignant Tumor Epigenetics and Gene Regulation, Sun Yat-Sen Memorial Hospital, Sun Yat-Sen University, Guangzhou, China 510120.

2. Department of Thoracic Surgery, Sun Yat-Sen Memorial Hospital, Sun Yat-Sen University, Guangzhou, China 510120

3. Department of Thoracic Surgery, Affiliated Cancer Hospital \& Institute of Guangzhou Medical University, Guangzhou, China 510120.

4. Breast Tumor Center, Sun Yat-Sen Memorial Hospital, Sun Yat-Sen University, Guangzhou, China 510120.

* These authors contributed equally to this work.

$\square$ Corresponding authors: Minghui Wang, Guangdong Provincial Key Laboratory of Malignant Tumor Epigenetics and Gene Regulation, Sun Yat-Sen Memorial Hospital, Sun Yat-Sen University, Guangzhou 510120, China; Department of Thoracic surgery, Sun Yat-Sen Memorial Hospital, Sun Yat-Sen University, Guangzhou 510120, China. Tel: +86-13826276828; Fax: +86-020-81332295; E-mail: wmingh@mail.sysu.edu.cn or Duoguang Wu, Guangdong Provincial Key Laboratory of Malignant Tumor Epigenetics and Gene Regulation, Sun Yat-Sen Memorial Hospital, Sun Yat-Sen University, Guangzhou 510120, China; Department of Thoracic surgery, Sun Yat-Sen Memorial Hospital, Sun Yat-Sen University, Guangzhou 510120, China. Tel: +86-13824471158; Fax: +86-020-81332295; E-mail: wudg3@mail.sysu.edu.cn

() The author(s). This is an open access article distributed under the terms of the Creative Commons Attribution License (https://creativecommons.org/licenses/by/4.0/). See http://ivyspring.com/terms for full terms and conditions.

Received: 2019.04.07; Accepted: 2019.09.08; Published: 2020.01.01

\begin{abstract}
Background: In microenvironment of malignant tumors, Hypoxia-Inducible Factors (HIF), most importantly HIF-1 a, play an important role in regulation of adaptive biological response to hypoxia, promoting angiogenesis and metastasis. However, the underlying mechanism that HIF-l $\alpha$ regulates metastasis needs to be further clarified.

Methods: The expressions of HIF-1 $1 \alpha$ and SPI were detected in 182 samples of esophageal squamous cell carcinoma (ESCC) and adjacent normal tissues by immunohistochemistry (IHC), and the correlation between the expression levels of HIF-1 $\alpha$ and SPI was analyzed. The expression of HIF-l $\alpha$ in ESCC cell lines TEl and KYSE30 was then detected using QRT-PCR and western blot. The potential binding sites of HIF-1 $\alpha$ on the SPI promoter were analyzed using UCSC and JASPAR databases, verified by chromosomal immunoprecipitation (ChIP) assay and qRT-PCR. The effects of HIF-I $\alpha$ and SPI on ESCC cell migration and invasion were then tested with Transwell and Matrigel experiments.

Results: The expression of HIF-l $\alpha$ in cancer tissues is higher than adjacent normal tissues, and is correlated with metastasis, recurrence and poor prognosis. Upon silencing HIF-1 $\alpha$ by siRNA, the invasion and migration ability of ESCC cells were significantly inhibited, which could be restored by the overexpression of SPI. Hypoxic conditions significantly increased the expression of HIF-1 $\alpha$ and SPI at both protein and mRNA levels in ESCC cells. HIF-1 a enhanced SPI transcription through binding to the promoter region. The expression of protein and mRNA levels of SPI was decreased by silencing HIF-l $\alpha$ in cells. In contrast, overexpression of HIF-1 $\alpha$ significantly increased the mRNA and protein levels of SPI. The expression of SPI in ESCC was positively correlated with the protein expression of HIF-l $\alpha$ and poor prognosis.

Conclusion: The results of our study indicate that HIF-1 a promotes metastasis of ESCC by targeting SPI in a hypoxic microenvironment. Further study on this mechanism may elucidate the possibility of HIF-I $\alpha$ and SPI as new targets for the treatment of ESCC.
\end{abstract}

Key words: HIF-1a, SP1, ESCC, tumor metastasis

\section{Introduction}

Esophageal squamous cell carcinoma (ESCC) is one of the most common malignant tumors of digestive tract in the world [1]. Based on histopathology, esophageal cancer is usually classified into squamous cell carcinoma and adenocarcinoma, with squamous cell carcinoma 
accounting for about $90 \%$ of patients in China [2]. Despite the continuous improvement in the diagnosis and treatment, the prognosis of patients with esophageal cancer remains poor, with the overall 5-year survival rate of $15 \%-34 \%$ [3, 4]. At present, surgery remains to be the major treatment of esophageal cancer. Combination with preoperative neoadjuvant or postoperative adjuvant radiotherapy and chemotherapy has improved patient prognosis at certain level, however the overall effects of treatment are still unsatisfactory. One of the major factors for poor prognosis of esophageal cancer patients is the tumor metastasis [5-7]. Searching of novel biomolecules related to tumor metastasis and clarification of underlying molecular mechanisms could facilitate the screening of patients with high risk of recurrence, as well as the identification of new therapeutic target, therefore would further improve the clinical treatment of esophageal cancer.

Similar to most solid tumors, insufficient oxygen supply often results in a hypoxic microenvironment in esophageal cancer tissue [8]. Hypoxia-Inducible Factors (HIF) are important regulators of adaptive response to hypoxia. As one of the most important regulators, elevated expression of HIF-1a has been identified in esophageal cancer $[7,9,10]$, prostate cancer [ 11], lung cancer [12], breast cancer [13] and other malignant tumors. It has been reported that HIF-1a could stimulate tumor angiogenesis and lymphangiogenesis, and promote metastasis and recurrence [14-17]. However, the exact functional mechanism of HIF-1a in metastasis of ESCC still needs to be further explored.

It has been reported that specific protein 1 (SP1) regulates the expression of various genes, including housekeeping genes as well as genes involved in regulation of cell proliferation, apoptosis, embryonic development and other physiological activities [18]. The expression and function of SP1 varies in different tumor cells. Multiple studies have confirmed the important regulatory role of SP1 in esophageal cancer [19], pancreatic cancer [20] and breast cancer [21]. HIF-1a functions as a transcription factor to regulate the expression of multiple genes [22-24]. Database prediction has shown that SP1 might be a potential target of HIF-1a's regulation, suggesting the function of HIF-1a in promoting tumor development and metastasis may through the regulation of SP1.

Studies of HIF-1a and SP1 in tumor metastasis are rare and related mechanisms remain unclear. This study showed that the HIF-1a protein level was higher in cancer tissues than in adjacent normal tissues, and the expression of HIF-1a was correlated with tumor metastasis, recurrence and poor prognosis in patients with esophageal cancer. In addition,
HIF-1a, bound to the SP1 promoter, regulated SP1 transcription, thereby inducing changes in migration and invasion abilities of esophageal cancer cells. There was a positive correlation between SP1 and HIF-1a protein expression in ESCC samples, and SP1 expression was also correlated with tumor metastasis, recurrence and poor prognosis. In conclusion, the study provided evidence for the molecular mechanism that HIF-1a promotes the metastasis of ESCC through targeting SP1 transcription. The results indicate the possibility for HIF-1a and SP1 as prognostic factors of ESCC.

\section{Materials and Methods}

\section{Clinical samples and data collection}

Cancer tissue specimens and paraffin sections of adjacent tissues were collected from 182 patients with ESCC who were treated with thoracic surgery at Sun Yat-Sen Memorial Hospital of Sun Yat-Sen University between January 2010 and January 2013. Diagnosis of ESCC for all patients were pathologically confirmed. No patient underwent chemotherapy or radiotherapy before surgery. The usage of patients' tissues was approved by the Ethics Committee of Sun Yat-Sen Memorial Hospital of Sun Yat-Sen University, and informed consents were acquired from all the patients. The clinical pathology and other clinical features of these patients were collected from electronic medical records.

\section{Immunohistochemistry}

Surgically removed cancer and metastatic lymph node tissues were immediately fixed in $10 \%$ formaldehyde, embedded in paraffin, and then sectioned. After dehydration with xylene and series of ethanol, samples were incubated in $3 \% \mathrm{H}_{2} \mathrm{O}_{2}$ for 10 min at room temperature, washed with PBS, incubated in antigen retrieval solution (sodium sulphate buffer $\mathrm{pH} \mathrm{6.0)}$ for high pressure retrieval, then naturally cooled to room temperature and washed with PBS. After blocking samples with 3\% bovine serum albumin for $15 \mathrm{~min}$, SPl antibody (rabbit anti-human, Abcam, USA, 1:100 dilution) was added and samples were incubated at $4{ }^{\circ} \mathrm{C}$ overnight. After rinsing samples with PBS, universal immunohistochemical secondary antibody (ZhongshanJinqiao, PV-6000, China) was added and samples were incubated for $30 \mathrm{~min}$ at $37^{\circ} \mathrm{C}$. After washing samples with PBS, substrate diaminobenzidine (DAB) was added and staining was controlled with regular microscopy. The samples were then counterstained with hematoxylin. After washing by water and decoloring by $1 \%$ hydrochloric acid ethanol, the samples were put into tap water for bluing. After dehydrating and transparentizing by series of ethanol 
and xylene, the specimens were sealed by neutral resins for observation.

The scoring of immunohistochemical staining of SP1 was determined using a double-blinded method. Each slice was observed and scored by two senior pathologists separately using a semi-quantitative scoring method. The effect that brown-yellow particles were observed in the nucleus or the cytoplasm was considered as SP1 positive. The samples were then scored according to the percentage of positive cells: 0 as lower than $10 \% ; 1$ as $10 \% \sim 25 \%$; 2 as $25 \% \sim 50 \% ; 3$ as $50 \% \sim 75 \%$; and 4 as $75 \%$ and above. The staining intensity of positive cells was categorized into 4 levels: no expression (0), weak (1), medium (2), and strong ( 3 points). The final score was the product of the percentage score and the stain intensity score. A final score of 0-3 was then assigned as low expression, and 4-12 was as assigned high expression (4-6: +, 7-9: ++, 10-12: +++).

\section{Cell line and culture}

The human ESCC cell line TE1 and KYSE30 was acquired from the Cell Bank of the Chinese Academy of Sciences (Shanghai, China). Cells were cultured in Roswell Park Memorial Institute (RPMI) 1640 supplemented with $10 \%$ fetal bovine serum (FBS) (Gibco, CA, USA). Cell culture plates were kept in a humidified incubator at $37^{\circ} \mathrm{C}$ with $5 \% \mathrm{CO}_{2}$. For the hypoxic treatment, cells were cultured in an oxygen-controlled incubator (Heal Force, Shanghai, China) under hypoxia conditions $\left(1 \% \mathrm{O}_{2}, 5 \% \mathrm{CO}_{2}\right.$ and $94 \% \mathrm{~N}_{2}$ ) for 24 to 48 hours.

\section{Total RNA extraction and RT-PCR}

Total RNA was extracted from ESSC cells using TRIzol Reagent (Invitrogen, Carlsbad, CA, USA). The cDNA was then reverse transcribed using a PrimeScriptTM RT kit with gDNA Eraser (TakaRa, Dalian, China). RT-PCR was performed using SYBR® PremixExTaqTMII (TakaRa, Dalian, China) on a LightCycler® 96SW 1.1 RT-PCR system (Roche, Basel, Switzerland) following the manufacturer's instructions. $\beta$-Actin was used as an internal control.

Primers for PCR are: HIF-1a, 5'- TGG CTG CAT CTC GAG ACT TT -3'(forward) and 5'- GAA GAC ATC GCG GGG AC -3'(reverse); SP1, 5'- ACC AAG CTG AGC TCC ATG AT -3'(forward) and 5'- CCT CAG TGC ATT GGG TAC TTC -3' (reverse); $\beta$-Actin, 5'-TCG TGC GTG ACA TTA AGG AG-3'(forward) and 5 '-GTC AGG CAG CTC GTA GCT CT-3' (reverse).

\section{Western blot}

Cells were lysed in RIPA buffer (Cell Signaling Technology) containing protease inhibitors. The lysate was centrifuged and the supernatant was collected.
Protein concentration was determined using a BCA

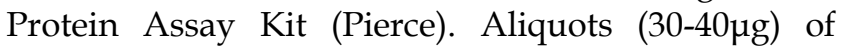
protein were separated on $10 \%$ SDS-PAGE electrophoresis gel and transferred to PVDF membrane (Immobilon-membrane, Millipore). After washed with Tris buffered saline Tween-20 buffer (TBST; pH 7.5; $10 \mathrm{mM}$ Tris, $150 \mathrm{mM} \mathrm{NaCl}$ and $0.1 \%$ Tween 20) for $10 \mathrm{~min} \times 3$ times, PVDF membrane was blocked in 5\% skim milk for 1 hour, then incubated overnight at $4{ }^{\circ} \mathrm{C}$ with primary antibodies (anti- $\beta$-ACTIN, anti-HIF-1a or anti-SP1; 1:1000, CST, USA). After washed three times with TBST ( $\mathrm{pH} 7.5)$ at room temperature, the PVDF membrane was incubated for 1 hour at room temperature with the secondary antibody, then washed three times with TBST ( $\mathrm{pH} 7.5)$ at room temperature for $10 \mathrm{~min} \times 3$ times. Proteins were then detected using a chemiluminescence and imaging system (Bio-Rad, USA).

\section{Transient transfection}

HIF-1a siRNA1,2 and negative control siRNA were synthesized by GenePharma (Shanghai, China). SP1 siRNA1,2 and negative control siRNA were synthesized by Ribobio (Ribobio Co., China). Cells were transfected with Lipofectamine 3000 (Invitrogen) according to the manufacturer's instructions. After 24 hours of hypoxia treatment, cells were transfected with HIF-1a siRNA for 12 hours. After 48 hours of transfection, cells were harvested and subjected to Western blot analysis.

The pc-HIF-1a plasmid was synthesized by Sino biological, and the pc-SP1 and pcDNA3.1 plasmids were constructed and synthesized by OBIO Technology (Shanghai). The pcDNA3.1 was used as an empty vector. Transfection was performed using Lipofectamine 3000 and P3000 (Invitrogen) according to the manufacturer's instructions. Cells were treated with plasmid for 12 hours and after 48 hours of transfection, cells were harvested and subjected to Western blot analysis.

\section{ChIP-PCR assays}

Formaldehyde ( $1 \%$ concentration) was added into cultured cells, and the cultured cells was then incubated at $37^{\circ} \mathrm{C}$ for $10 \mathrm{~min}$. The cells were then washed twice with PBS, collected and treated with TNT lysate solution $(20 \mathrm{mmol} / \mathrm{L}$ Tris- $\mathrm{HCl}, \mathrm{pH}=7.4$, $200 \mathrm{mmol} / \mathrm{L} \mathrm{NaCl}, 1 \%$ Triton X-100, $1 \mathrm{mmol} / \mathrm{L}$ PMSF and $1 \%$ aprotinin) in ice bath. The DNA was sheared into 200-500 bp fragments with ultrasonication and co-immunoprecipitated with $2 \mu \mathrm{g}$ TS2 antibody. Antibody/protein complexes were collected using Protein A magnetic beads and washed 3 times with ChIP buffer (5\% SDS, $1 \mathrm{mmol} / \mathrm{L}$ EDTA, $0.5 \%$ bovine 
serum albumin and $40 \mathrm{mmol} / \mathrm{L} \mathrm{NaHPO} 4, \mathrm{pH}=7.2$ ). After $1 \%$ SDS and $1 \mathrm{mmol} / \mathrm{L} \mathrm{NaHCO} 3$ were used to wash the immunoprecipitant, $200 \mathrm{mmol} / \mathrm{L} \mathrm{NaCl}$ and RNaseA were added to the immunoprecipitant, and the immunoprecipitant was incubated at $65^{\circ} \mathrm{C}$ for $4 \mathrm{~h}$ to de-crosslink. The samples were then treated with proteinase $\mathrm{K}$ for $2 \mathrm{~h}$, and DNAs were purified using chormatography. The SP1 promoter DNA was detected using PCR amplification. Primers used to amplify the -401 to -142 region of $S \mathrm{p} 1$ promoter are: Forward: 5'-CAGCAAGTCACTCCC-3'; Reverse: 5'-GGAGAGGTGCGCGGC-3'. RT-PCR was performed for samples in 3 groups: IgG group (negative control), Input group (positive control), and IP group.

\section{Cell migration and invasion detection}

Cell migration and invasion were assayed in triplicate using 24-well transwell plates and polycarbonate nucleus filters (Merck Millipore Bioscience, Germany) with 8- $\mu \mathrm{m}$ pore size. The cells were deprived of FBS for 24 hours before each experiment. For the invasion assay, inserts were pre-coated with Matrigel (BD Biosciences, Belgium) diluted 1:10 in serum-free Ham's F10 and Matrigel was allowed to polymerize at $37^{\circ} \mathrm{C}$ for 1 hour. The inserts were not coated in the migration assay. Control and treated cells $\left(1 \times 10^{5}\right.$ in $200 \mu$ serum free medium) were seeded in the upper chamber of the insert. The lower chamber was filled with $600 \mu \mathrm{l}$ of medium containing $20 \%$ FBS as an attractant. The invasion assay was allowed to proceed for 24 hours and migration assay was incubated for 12 hours. Any cells remaining on top of the insert are scraped off by scraping. The migrating cells attached to the lower side were fixed in methanol for 10 minutes and stained with $0.05 \%$ crystal violet for 20 minutes. The cells were observed under a microscope and cell numbers in 3 random fields in the middle of the membrane were counted.

\section{Statistical methods}

Statistical analysis was performed using SPSS 20.0 statistical software. All data were tested for normality. Quantitative data with normal distribution was represented as mean \pm SD of triplicate samples and differences between groups were tested using t-test and the analysis of variance (ANOVA). The Mann-Whitney $U$ test was used for the comparisons of skew distributed data. The Chi-square test was used for the comparisons of categorical data between groups. Correlation between variables was analyzed using Pearson correlation analysis. Kaplan-Meier analysis and Log-rank test were used to assess differences in patient survival. All statistical tests were two-sided tests, and $\mathrm{p}<0.05$ was considered as statistically significant.

\section{Results}

\section{HIF-1 $\alpha$ promotes the metastasis of esophageal cancer}

For the study of HIF-1a functions in esophageal cancer development, samples of cancer tissues and adjacent normal tissues were collected from 182 patients with ESCC. The results of immunohistochemistry assays showed that the expression of HIF-1a protein was significantly higher in ESCC than in adjacent normal tissues (Figure 1a and Table 1). In addition, the expression of HIF-1a in cancer tissues was associated with metastasis and recurrence (Table 2). Kaplan-Meier analysis showed that compared to those with lower HIF-1a expression, the patients with higher HIF-1a expression have higher rates of early metastasis and recurrence (estimated 3-year recurrence rate70.83\% vs $45.46 \%$, Figure $1 \mathrm{~b}$ ) and lower survival (estimated 5-years survival rate $8.15 \%$ vs $20.25 \%$, Figure 1c).

Table 1. The expression of HIF-1 $\alpha$ in ESCC and adjacent normal tissues

\begin{tabular}{llllll}
\hline ESCC & Case n & \multicolumn{2}{l}{ Expression of HIF-1a } & \multirow{2}{*}{$\boldsymbol{X}^{2}$ value } & P value \\
\cline { 3 - 4 } & & Low & High & & \\
\hline Tumor tissues & 182 & 80 & 102 & 87.72 & $<0.001$ \\
$\begin{array}{l}\text { Adjacent } \\
\text { normal tissues }\end{array}$ & 182 & 164 & 18 & & \\
\hline
\end{tabular}

Table 2. Clinical characteristics and its relationship with HIF- $\alpha$ / Spl expression

\begin{tabular}{lllll}
\hline Clinical characteristic & $\begin{array}{l}\text { HIF-1a high } \\
\text { expression }\end{array}$ & $\mathbf{p}$ value & $\begin{array}{l}\text { Sp1 high } \\
\text { expression }\end{array}$ & p value \\
\hline Gender & $91 / 161$ & 0.82 & & 0.10 \\
$\begin{array}{l}\text { Male } \\
\text { Female }\end{array}$ & $11 / 21$ & & $81 / 161$ & \\
Age & & 0.18 & $15 / 21$ & \\
$\geq 60$ & $44 / 87$ & & $44 / 87$ & 0.66 \\
$<60$ & $58 / 95$ & 0.40 & $52 / 95$ & \\
$\begin{array}{l}\text { Invasion depth } \\
\text { T1 }\end{array}$ & $5 / 13$ & & $3 / 13$ & 0.06 \\
T2 & $33 / 59$ & & $30 / 59$ & \\
T3+T4a & $64 / 110$ & & $63 / 110$ & \\
Lymphatic metastasis & & 0.023 & & $<0.001$ \\
yes & $68 / 108$ & & $69 / 108$ & \\
no & $34 / 74$ & & $27 / 74$ & \\
$\begin{array}{l}\text { Differentiation } \\
\text { well }\end{array}$ & $37 / 55$ & 0.12 & & 0.88 \\
moderate & $31 / 63$ & & $28 / 55$ & \\
low & $34 / 64$ & & $32 / 63$ & \\
Recurrence & & 0.045 & $30 / 64$ & \\
yes & $55 / 88$ & & $59 / 88$ & \\
no & $27 / 59$ & & $24 / 59$ & \\
\hline
\end{tabular}


The migration and invasion ability of tumor cells is critical for tumor metastasis. In further analysis of the effect of HIF-1a on the migration and invasion of ESCC cells, it was found that the migration and invasion of ESCC cells were significantly inhibited by siRNA silencing of HIF-1a (Figure 1d). In contrast, significantly enhanced cell migration and invasion were observed upon overexpression of HIF-1a (Figure 1e).

These results indicated that HIF-1a might play an important role in metastasis, recurrence and poor prognosis of ESCC patients.

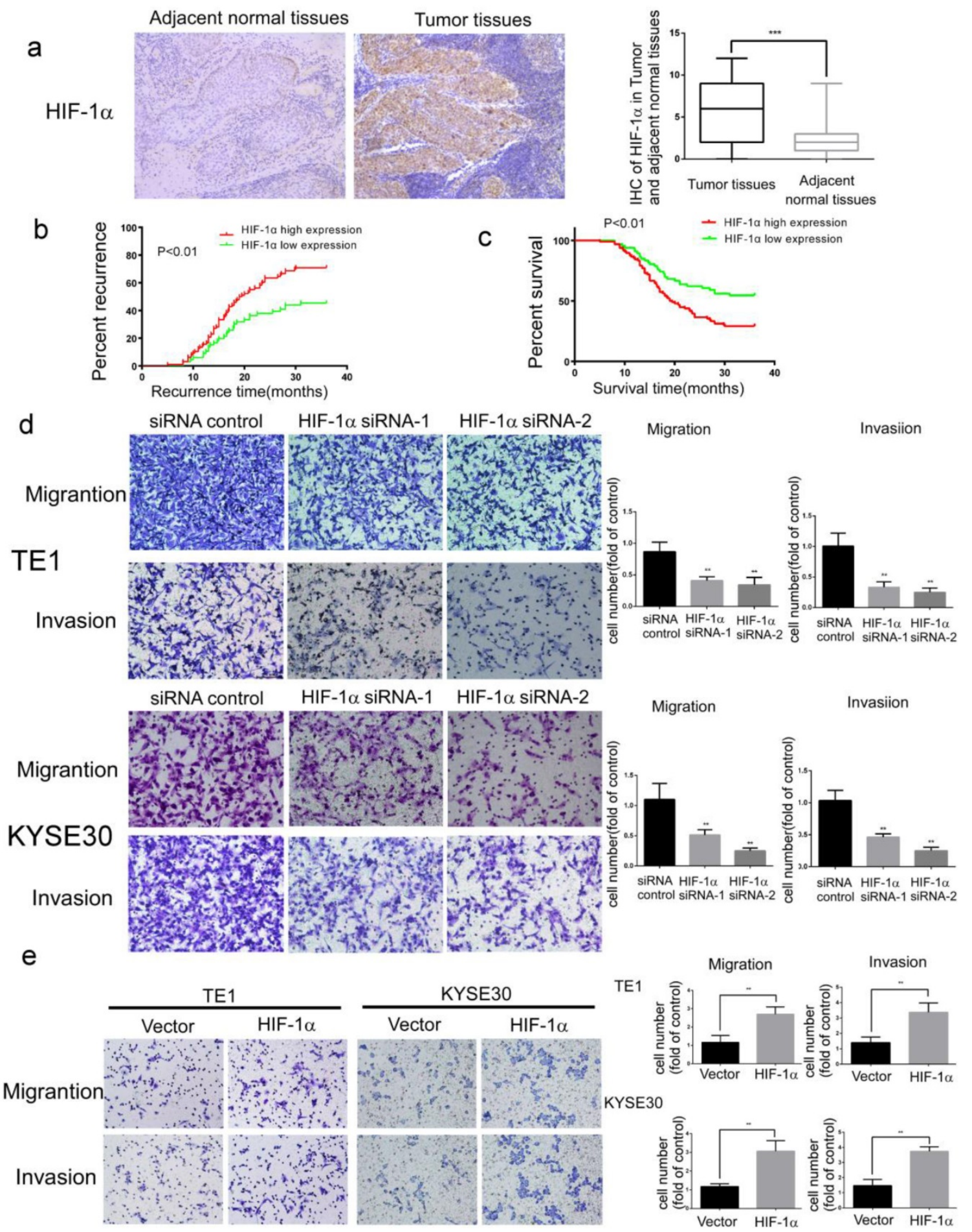

Figure 1. The expression of HIF-1 $\alpha$ in ESCC tissues and the functions of HIF-1 $\alpha$ in ESCC cells. a The expression of HIF-1 $\alpha$ in ESCC detected by IHC, and the immunoreactivity score of HIF-1 $\alpha$ in ESCC tumor tissues and adjacent normal tissues $(n=182)$; $b$ the 3-year regional recurrence curve of patients with HIF-1 $\alpha$ high/low expression; $\mathbf{c}$ the 5 -year survival curve of patients with HIF-la high/low expression; $\mathbf{d}$ silencing HIF-la by siRNA significantly suppressed the migration and invasion of TE1 and KYSE30, $(n=3)$. Data represent the mean \pm S.D; e overexpression of HIF- $/ \alpha$ promoted migration and invasion of TE1 and KYSE30, $(n=3)$. Data represent the mean \pm S.D. $(* * \mathrm{P}<0.01, * * * \mathrm{P}<0.001)$. 


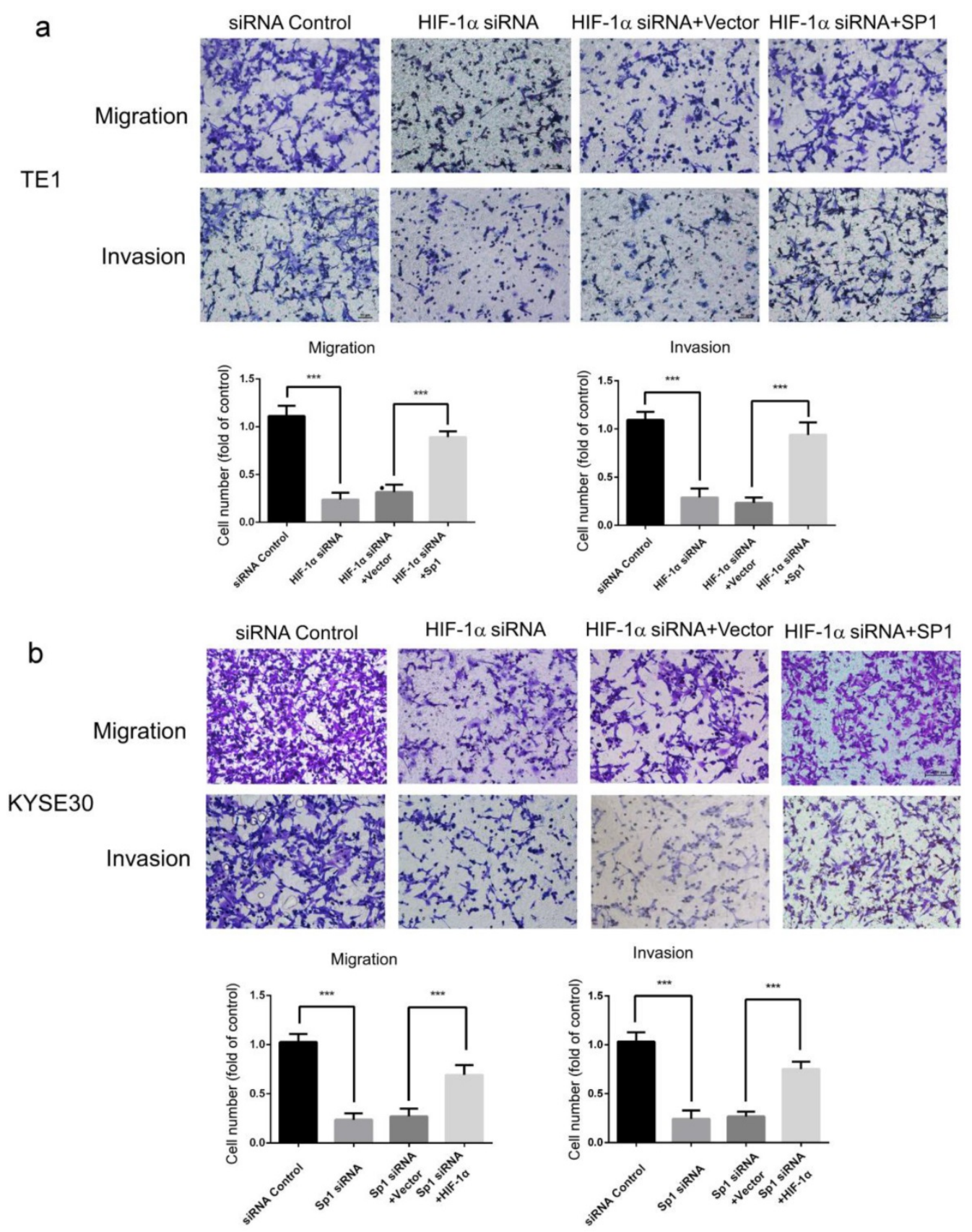

Figure 2. Effects of SPI overexpression on the migration and invasion of hypoxic ESCC cells. a, b migration and invasion of TEl and KYSE30 cells can be blocked by HIF-1 $\alpha$ silencing, and can be rescued by SPI overexpression $(n=3)$. Data represent the mean \pm S.D. $(* * * *<0.001)$.

\section{HIF-1a promoted migration and invasion of ESCC cells by targeting Sp 1}

The above results confirmed that HIF-1a is an essential factor for the development of ESCC. It has been reported that HIF-1a regulates the expression of various target genes as transcription factor, therefore it is likely that its function in promoting metastasis of esophageal cancer is also working through the activation of downstream target gene transcription.

Potential downstream target genes of HIF-1a were predicted in the UCSC and JASPAR databases and predicted candidate genes were further screened for those closely related to metastasis of ESCC. Among these genes, SP1 has been reported to be involved in multiple mechanisms related to metastasis of ESCC [25-28]. So we suspect that HIF-1a may regulate migration and invasion through SP1. The role of SP1 in HIF-1a-regulated migration and invasion of esophageal cancer cells were then investigated using complementation assays. The results showed that overexpression of SP1 significantly restored the migration and invasion of ESCC cells which was inhibited by HIF-1a silencing (Figure 2ab). These results indicated that HIF-1a 
regulated the migration and invasion of esophageal cancer cells by targeting SP1, and SP1 may be a critical factor in the regulation mechanism of HIF-1a.

\section{HIF-1 a regulates SPI expression through directly binding to the SPI promoter}

Next, we further studied the molecular mechanism of HIF-1a regulating SP1 expression. Forecast by UCSC and JASPAR database, A possible binding site (Figure 3a) of HIF-1a was identified in the SP1 promoter (-1246 to $-1237 \mathrm{bp})$. In subsequent Chip-PCR assay performed with extracts from ESCC cell lines, significant enrichment of SP1 promoter sequence was achieved through immunoprecipitation with anti-HIF-1a antibody, but not with control IgG (Figure 3bc). These results indicated that as a transcription factor, HIF-1a could directly bind to the promoter of SP1. The regulatory effect of HIF-1a on SP1 was then verified in the esophageal cancer cell TE-1. The expression of HIF-1a and SP1 was firstly monitored under hypoxic conditions (48h). The results of qRT-PCR and Western blot showed that there was no significant change in the transcripts of HIF-1a in hypoxic condition, but the protein level was enhanced, this result is consistent with the literature [29]. While, both the transcription and protein level of SP1 were increased significantly (Figure 3de). These data indicated that hypoxia enhances protein expression of HIF-1a and SP1 in ESCC cells. Upon siRNA silencing of HIF-1a, the mRNA levels of HIF-1a and SP1 were significantly decreased (detected with qRT-PCR, Figure 3f), as well as the protein levels (detected with Western blot, Figure 3g). Upon overexpression of HIF-1a, the mRNA and protein levels of SP1 were significantly increased (Figure 3h). These data indicated that HIF-1a could directly regulate the transcriptional level of $S P 1$, therefore affect its protein expression level.

\section{Expressions of HIF- $1 \alpha$ and SPI are positively correlated, and associated with metastasis, recurrence as well as poor prognosis.}

To elucidate the relation between HIF-1a and SP1 in ESCC tissues, we examined the expression of SP1 in 182 ESCC tissues and adjacent normal tissues by immunohistochemistry. It was found that the expression of SP1 was higher in the ESCC tissues than in adjacent normal tissues (Figure 4a, Table 3). As shown in Table 2, high expression level of SP1 was significantly associated with tumor metastasis and recurrence (Table 2). Kaplan-Meier analysis estimated that the 3-year recurrence rate in patients with positive and negative expression of SP1 was $72.04 \%$ and $44.93 \%$, respectively (Figure $4 \mathrm{~b}$ ), and the 5-year survival rate in patients with positive and negative expression of SP1 was estimated as $6.385 \%$ and $22.382 \%$, respectively (Figure 4c). The immunohistochemical score of SP1 (Figure 4d) was significantly higher in HIF-1a high expression group $(8.887 \pm 2.170)$ than in HIF-1a low expression group $(5.913 \pm 2.531)$. There was a positive correlation between the expression of HIF-1a and SP1 in esophageal cancer tissues $(\mathrm{R}=0.5388 \mathrm{P}<0.0001$, Figure 4e).

Among 108 ESCC patients with tumor metastasis, 68 patients $(62.9 \%)$ had high expression of HIF-1a, 69 patients $(63.9 \%)$ had high expression of SP1; while among 74 patients without tumor metastasis, 34 patients $(45.9 \%)$ were with high expression of HIF-1a and 27 patients (36.5\%) had high expression of SP1 (Figure 4f). Both HIF-1a and SP1 were positively associated with tumor metastasis $(\mathrm{P}<0.001$, Table 2). Among the 108 patients with tumor metastasis, 56 patients $(51.8 \%)$ had high expression of both HIF-1a and SP1, 12 patients (11.1\%) had high expression of HIF-1a, 13 patients $(12.0 \%)$ had high expression of SP1, and 27 patients (25.0\%) patients had low expression of both HIF-1a and SP1 (Figure 4g). Patients with high expression of both HIF-1a and SP1 were more likely to have tumor metastasis (Table 4).

Kaplan-Meier analysis estimated that patients with high expression of both HIF-1a and SP1 had a higher 3-year recurrence rate of $75.0 \%$ (Figure $4 \mathrm{~h}$ ), and lower 5-year survival rate of $12.11 \%$ (Figure $4 \mathrm{i}$ ), compared with the others.

The above results indicated that there was a positive correlation between HIF-1a and SP1 levels in esophageal cancer tissues, and concurrent expressions of these two proteins were associated with poor prognosis.

\section{Discussion}

ESCC is a highly invasive tumor. Metastasis is the major-risk factor affecting the prognosis of ESCC patients, especially Lymph node metastasis, with metastasis as the most common cause of surgical treatment failure. Therefore, research in tumor metastasis mechanism of esophageal cancer is of great significance for the exploration of novel target to improve treatment effect and prognosis of esophageal cancer patients. However, the related mechanism about metastasis of esophageal cancer is still unclear.

Table 3. The expression of SPI in ESCC and adjacent normal tissues

\begin{tabular}{llllll}
\hline ESCC & Case & \multicolumn{2}{c}{ Expression of Sp1 } & $\boldsymbol{X}^{2}$ value & P value \\
& $\mathbf{n}$ & Low & High & & \\
\hline Tumor tissues & 182 & 86 & 96 & 95.64 & $<0.001$ \\
Adjacent normal tissues & 182 & 171 & 11 & & \\
\hline
\end{tabular}


a

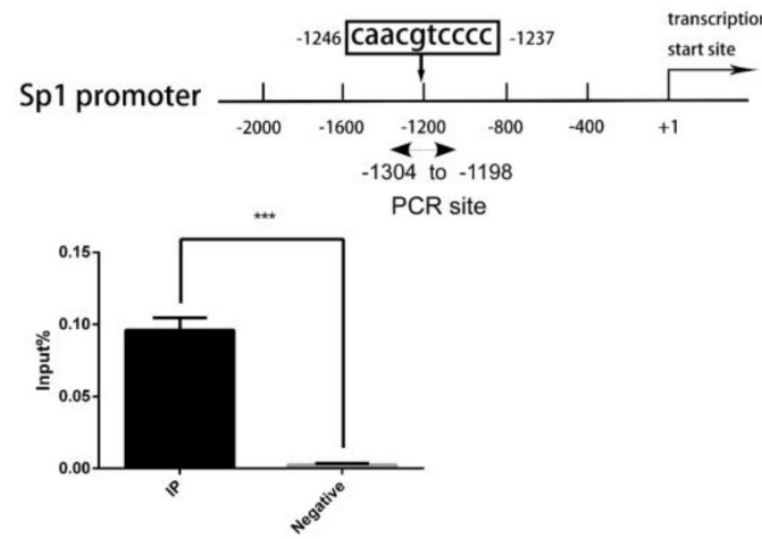

d

TE1

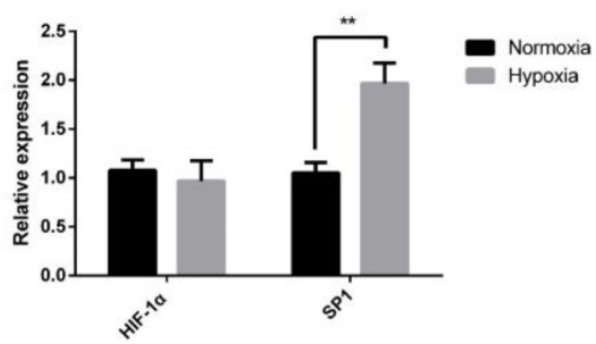

e

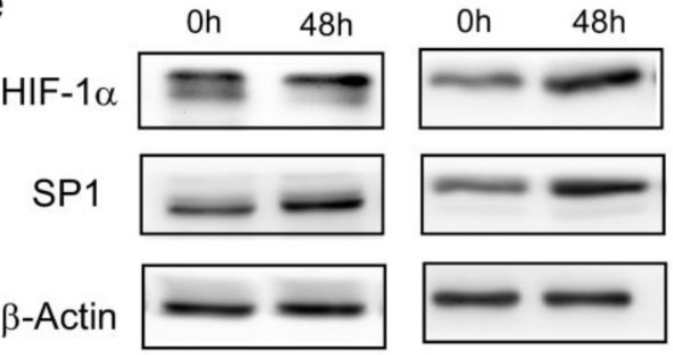

b M: DNA Ladder 500 Plus
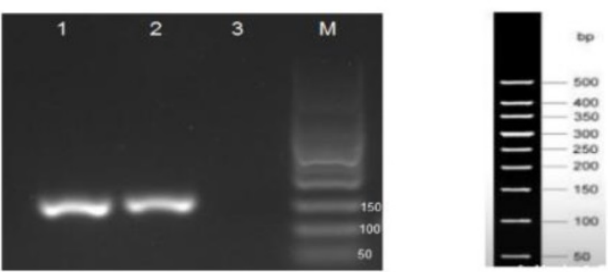

Lane 1: qPCR Amplification of Cell protein sample (chip) Input Lane 3: aPCR Amplification of Cell protein sample (chip) neg

KYSE30

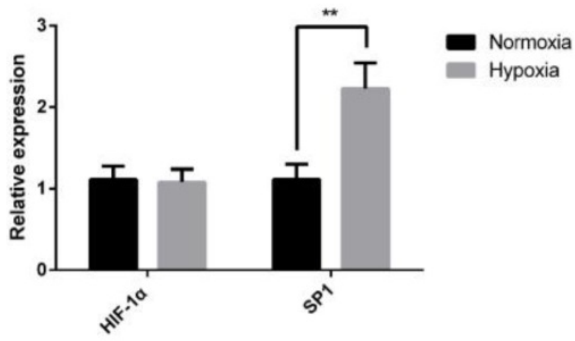

f

TE1

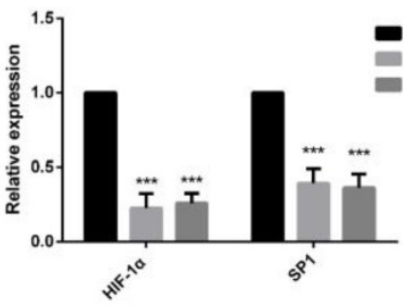

IRNA Control

HIF-1 $\alpha$ SIRNA-1 HIF-1 1 SIRNA-2

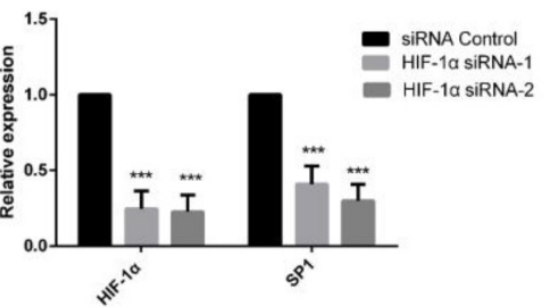

g

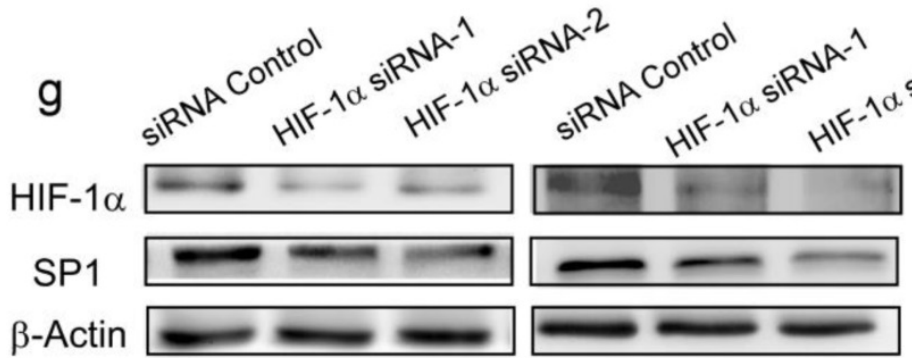

$\mathrm{h}$

Vector HIF-1 $\alpha$

Vector HIF-1 $\alpha$

HIF-10
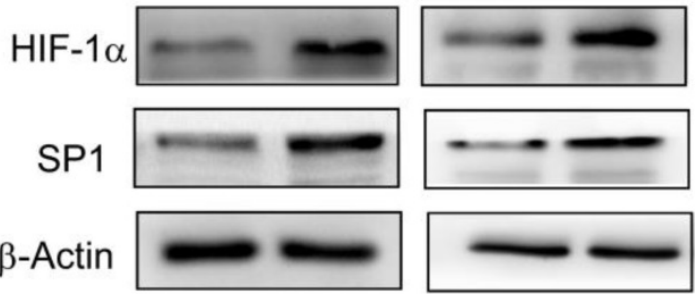

\section{$\beta$-Actin}

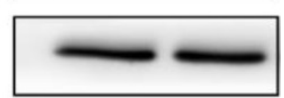

Figure 3. HIF-1 $\alpha$ up-regulates the protein expression of SPI and its transcriptional activity by directly binding to the SP1 promoter. a Schematic diagram depicting the positions of the primers used for the ChIP assay; $\mathbf{b}, \mathbf{c}$ ChIP analysis was performed using a negative control immunoglobulin $\mathrm{G}$ (IgG) or antiHIF-1 $\alpha$ antibody in TEl cells. Input positive control (anti- ARRDC3 antibodies); d HIF-1 $\alpha$ and SPI mRNA levels under both normoxic and hypoxic conditions were analyzed by real-time PCR. $\beta$-Actin was used as the internal control; e HIF-1 $\alpha$ and SP1 protein levels under both normoxic and hypoxic conditions(48h) were analyzed by Western blotting. $\beta$-Actin was used as the internal control; $\mathbf{f}$ a knockdown efficiency of HIF-I $\alpha$ siRNA, and the mRNA levels of SPI were detected by real-time PCR. $\beta$-Actin was used as the internal control $(n=3)$; $g$ a knockdown efficiency of HIF-l $\alpha$ siRNA, and the protein levels of SPI were analyzed by Western blotting. $\beta$-Actin was used as the internal control $(n=3)$; $\boldsymbol{h}$ the efficiency of over expression of HIF-I $\alpha$, and the protein levels of SPI were analyzed by Western blotting, the mRNA levels of SPI were detected by real-time PCR. $\beta$-Actin was used as the internal control $(n=3)$. $(* * P<0.01, * * * P<0.001)$. 
a

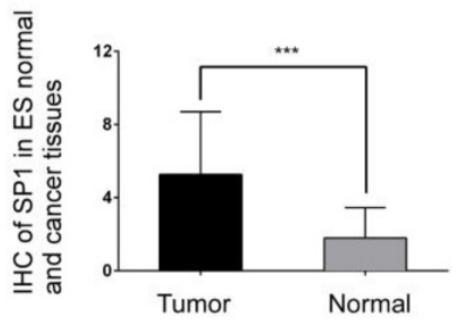

b

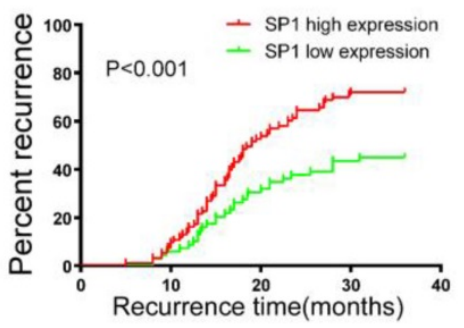

C

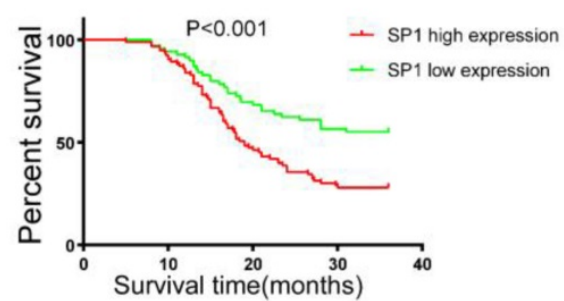

d

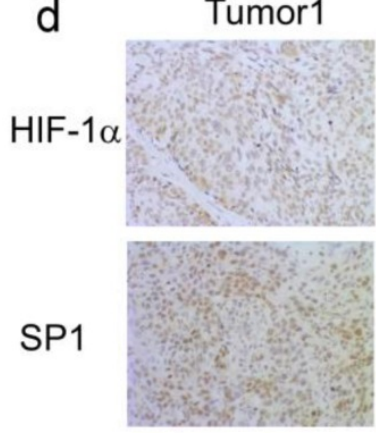

Tumor2
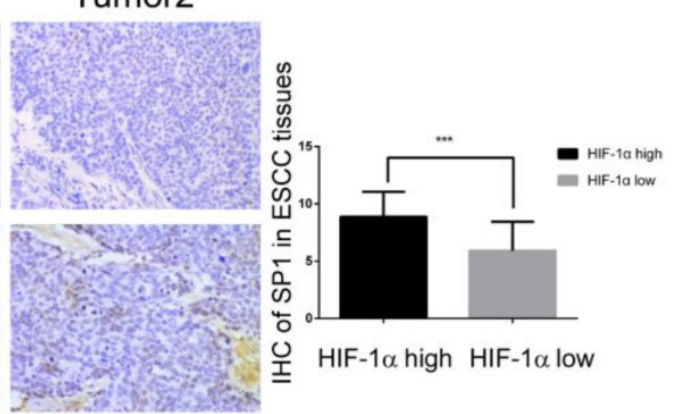

g
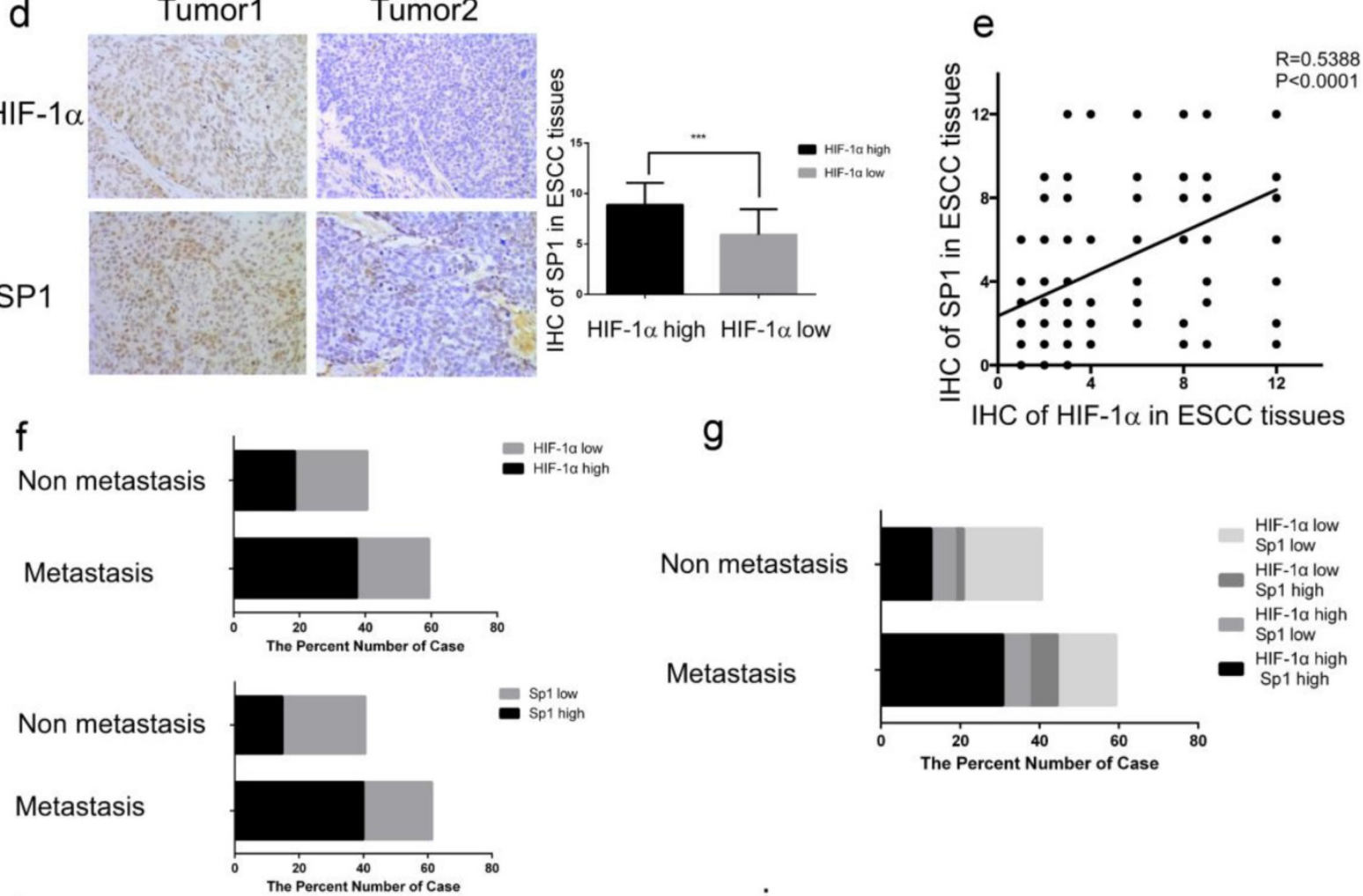

h
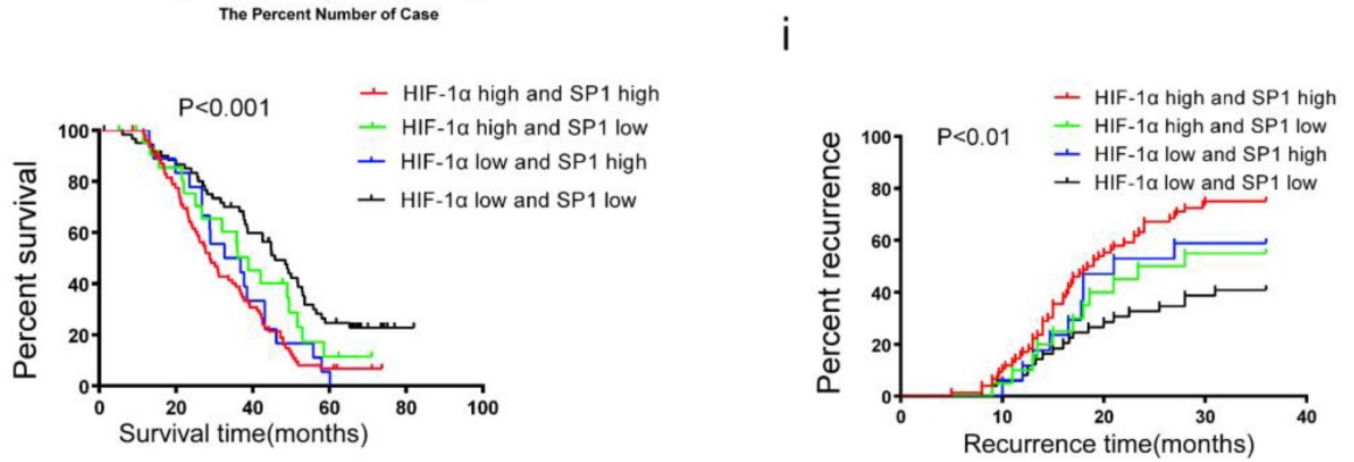

Figure 4. Co-expression of HIF-1 $\alpha$ and SPI in ESCC tissues. a The expression of SPI in ESCC tissues detected by IHC, the immunoreactivity score of SPI in ESCC tumor tissue and adjacent normal tissue $(n=182)$; $\mathbf{b}$ the 3-year regional recurrence curve of patients with SP1 high/low expression; $\mathbf{c}$ the 5 -year survival curve of patients with SPI high/low expression; d the expression of HIF-la and SPI in ESCC detected by IHC, the immunoreactivity score of SPI in HIF-1a high expression $(n=102)$ and HIF-1 $\alpha$ low expression $(n=80)$ groups; e the correlation between the expression of HIF-1 $\alpha$ and SP1 in esophageal cancer tissues; $\mathbf{f}, \mathbf{g}$ the percent number of high/low expression of HIF-l $\alpha$ and SPI in metastatic and non-metastatic groups; $\mathbf{h}$ the 5 -year survival curve of patients with HIF-1 $\alpha$ and SPI positive/negative expression; i the 3-year regional recurrence curve of patients with HIF-1 $\alpha$ and SPI positive/negative expression. (** $\mathrm{P}<0.01$, $* * * \mathrm{P}<0.001$ ).

Studies till present indicate that the main cause of metastasis of ESCC is the generation of blood vessels and lymphatics in the tumor microenvironment, which in turn promotes the metastasis of tumor cells to lymph nodes and distant organs [30-32]. HIF-1a is activated in hypoxic environment, and regulates transcription of downstream genes involved in various cell functions, 
including proliferation, survival, cell migration, glucose metabolism, $\mathrm{pH}$ regulation, angiogenesis and lymphangiogenesis, allowing cells to adapt to hypoxic environment, contributing to proliferation and highly invasive and metastatic ability [33-36]. The hypoxic tumor microenvironment induces increase in the transcriptional activity of HIF-1a, which in turn stimulates transcription of some genes associated with angiogenesis and lymphangiogenesis [30, 31]. Although it has been observed that the density of blood vessels and lymphatics is associated with high expression of HIF-1a in different malignant tumors, the prognostic value of these features in tumors from squamous epithelium is still controversial [37]. This study validated the relationship between HIF-1a expression and tumor metastasis in ESCC and the effect of HIF-1a on the prognosis of patients with ESCC.

Table 4. Clinical characteristics and its relationship with HIF- $\alpha$ \& Spl expression

\begin{tabular}{|c|c|c|c|c|c|}
\hline $\begin{array}{l}\text { Clinical } \\
\text { characteristic }\end{array}$ & $\begin{array}{l}\text { HIF-1a high } \\
\text { Sp1 high }\end{array}$ & $\begin{array}{l}\text { HIF-1a high } \\
\text { Sp1 low }\end{array}$ & $\begin{array}{l}\text { HIF-1a low } \\
\text { Sp1 high }\end{array}$ & $\begin{array}{l}\text { HIF-1a } \\
\text { low } \\
\text { Sp1 low }\end{array}$ & p value \\
\hline \multicolumn{5}{|l|}{ Gender } & 0.06 \\
\hline Male & $69 / 161$ & $22 / 161$ & $12 / 161$ & $58 / 161$ & \\
\hline Female & $10 / 21$ & $1 / 21$ & $5 / 21$ & $5 / 21$ & \\
\hline \multicolumn{5}{|l|}{ Age } & 0.36 \\
\hline$\geq 60$ & $36 / 87$ & $8 / 87$ & $8 / 87$ & $35 / 87$ & \\
\hline$<60$ & $43 / 95$ & $15 / 95$ & $9 / 95$ & $28 / 95$ & \\
\hline \multicolumn{5}{|c|}{ Invasion depth } & 0.22 \\
\hline $\mathrm{T} 1$ & $2 / 13$ & $3 / 13$ & $1 / 13$ & $7 / 13$ & \\
\hline $\mathrm{T} 2$ & $27 / 59$ & $6 / 59$ & $3 / 59$ & $23 / 59$ & \\
\hline $\mathrm{T} 3+\mathrm{T} 4 \mathrm{a}$ & $50 / 110$ & $14 / 110$ & $13 / 110$ & $33 / 110$ & \\
\hline \multicolumn{5}{|c|}{ Lymphatic metastasis } & 0.003 \\
\hline yes & $56 / 108$ & $12 / 108$ & $13 / 108$ & $27 / 108$ & \\
\hline no & $23 / 74$ & $11 / 74$ & $4 / 74$ & $36 / 74$ & \\
\hline \multicolumn{5}{|c|}{ Differentiation } & 0.21 \\
\hline well & $28 / 55$ & $9 / 55$ & $4 / 55$ & $14 / 55$ & \\
\hline moderate & $26 / 63$ & $5 / 63$ & $4 / 63$ & $28 / 63$ & \\
\hline low & $25 / 64$ & $9 / 64$ & $9 / 64$ & $21 / 64$ & \\
\hline \multicolumn{5}{|l|}{ Recurrence } & 0.019 \\
\hline yes & $40 / 88$ & $15 / 88$ & $12 / 88$ & $21 / 88$ & \\
\hline no & $22 / 59$ & $5 / 59$ & $4 / 59$ & $28 / 59$ & \\
\hline
\end{tabular}

Studies have shown that HIF-1a is associated with tumor metastasis in thyroid papillary carcinoma [38]. T.Kurokawa et al [7] showed that high expression of HIF-1a was positively correlated with tumor metastasis and poor prognosis in patients with ESCC. This was consistent with our results and indicated that HIF-1a plays an important role in metastasis of ESCC, although the molecular mechanism remains to be further clarified. In this study, we found that patients with high expression of both HIF-1a and SP1 in ESCC tissues were with higher recurrence rate and lower survival rate, this suggests that HIF-1a plays an important role in the metastasis and recurrence of ESCC with SP1.

Previous studies have confirmed that SP1 is highly expressed in gastric cancer, pancreatic cancer, etc. [39-41], related to high level of tumor malignancy and poor prognosis, which could accelerate tumor metastasis, promote epithelial-mesenchymal transition and regulate cell cycle [42, 43]. However, there still remains controversy about the functions of SP1, some reports expound that SP1 plays an important role in anticancer [44-46]. So, it is still unclear whether SP1 plays a dual role in the development of cancer, which requires further investigation. We just suspect that SP1 may change its transcriptional activity at different tumor stages. In this study, we found that SP1 is highly expressed in ESCC, and accelerates tumor metastasis.

At present, there are few studies on the mechanism of SP1 in metastasis of ESCC. Studies on the potential interaction mechanism between HIF-1a and SP1 have been reported in human glioblastoma [47] and CNS ischemia in rat [48]. However, it is unclear whether there is a specific interaction between HIF-1a and SP1 promoter in tumor cells.

In this study, we analyzed the UCSC and JASPAR database and found that HIF-1a may bind to the SP1 promoter region (-1246 to $-1237 \mathrm{bp})$ and verified the binding using ChIP assay. It was also proved that HIF-1a could directly regulate the expression of SP1 by binding to the promoter of SP1, thereby affecting the metastasis of ESCC. The concurrent high expressions of HIF-1a and SP1 were found to be associated with metastasis of ESCC $(\mathrm{P}<0.01$ Table 4$)$.

Activation of VEGF-C and VEGF-A in tumor microenvironment is critical for lymphangiogenesis and angiogenesis $[48,50]$. Studies have shown that HIF-1a could promoted the expression of VEGF-A [51], and it has been reported that SP1 could promote the expression of VEGF-C in breast cancer [52]. These results suggested that HIF-1a/SP1/VEGF regulatory pathway might be an important molecular mechanism in the metastasis of tumors.

In summary, this study has found that HIF-1a and SP1 are highly expressed in esophageal cancer tissues and the expression levels of these two are positively correlated. Concurrent high expression of these two proteins is closely related to tumor metastasis and poor prognosis. It is also confirmed that HIF-1a has a direct regulatory effect on the transcription of SP1 in esophageal cancer. Our results may help elucidate the molecular mechanism of tumor metastasis and recurrence in ESCC and provide a new theoretical basis for targeted therapy. 


\section{Acknowledgements}

This work was supported by grants from The National Natural Science Foundation of China (No. 81871886). Grant from The Natural Science Foundation of Guangdong Province, China (No. 2017A030313474). Grant from Guangdong Science and Technology Department (2017B030314026).

\section{Authorship}

Minghui Wang: Conceptualization, Project Administration, Funding Acquisition. Duoguang Wu: Supervision, Methodology, Writing - Review \& Editing. Xueting Hu: Writing - Original Draft, Data Curation, Software. Jiatong Lin: Formal Analysis, Data Curation. Ming Jang: Visualization. Xiaotian He: Resources. Wenjian Wang: Validation. Chuwen Hu: Investigation. Zhiwen Shen: Data Curation. Kefeng Wang: Formal Analysis. Huayue Lin: Visualization. Zhanghai He: Data Curation.

\section{Competing Interests}

The authors have declared that no competing interest exists.

\section{References}

1. Fitzmaurice C, Allen C, Barber RM, et al. Global, regional, and national cancer incidence, mortality, years of life lost, years lived with disability, and disability-adjusted life-years for 32 cancer groups, 1990 to 2015: a systematic analysis for the global burden of disease study. JAMA Oncol. 2017; 3: 524-8.

2. Torre LA, Bray F, Siegel RL, et al. Global cancer statistics, 2012. CA Cancer J Clin. 2015; 65: 87-108.

3. Sjoquist KM, Burmeister BH, Smithers BM, et al. Survival after neoadjuvant chemotherapy or chemoradiotherapy for resectable oesophageal carcinoma: an updated meta-analysis. Lancet Oncol. 2011; 12: 681-92.

4. Fakhrian K, Ordu AD, Lordick F, et al. Long-term outcomes of trimodality treatment for squamous cell carcinoma of the esophagus with cisplatin and/or 5-FU. StrahlentherOnkol. 2014; 190: 1133-40.

5. Mariette C, Piessen G, Triboulet JP. Therapeutic strategies in oesophageal carcinoma: Role of surgery and other modalities. The Lancet Oncology. 2007; 8:545-53.

6. Chen G, Wang Z, Liu XY, et al. Recurrence Pattern of Squamous Cell Carcinoma in the Middle Thoracic Esophagus after Modified Ivor-Lewis Esophagectomy. World J Surg. 2007; 31: 1107-14

7. Kurokawa $\mathrm{T}$, Miyamoto $\mathrm{M}$, Kato $\mathrm{K}$, et al. Overexpression of hypoxia-inducible-factor $1 \mathrm{a}(\mathrm{HIF}-1 \mathrm{\alpha})$ in oesophageal squamous cell carcinoma correlates with lymph node metastasis and pathologic stage. Br J Cancer. 2003; 89: 1042-7.

8. Pouyssegur J, Dayan F, Mazure NM. 2006. Hypoxia signalling in cancer and approaches to enforce tumour regression. Nature. 2006; 441: 437-43.

9. Corti L, Skarlatos J, Gatter K, et al. Hypoxia inducible factor (HIF-1a and HIF-2a) expression in early esophageal cancer and response to photodynamic therapy and radiotherapy. Cancer Res. 2001; 61: 1830-2.

10. Peerlings J, Lien VDV, Mitea C, et al. Hypoxia and hypoxia response-associated molecular markers in esophageal cancer: A systematic review. Methods. 2017; 130: 51-62.

11. Fraga A, Ribeiro R, Coelho, André, et al. Genetic polymorphisms in key hypoxia-regulated downstream molecules and phenotypic correlation in prostate cancer. BMC Urol. 2017; 17: 12

12. Berezowska S, Galván, José A, Langer R, et al. Glycine decarboxylase and HIF-1a expression are negative prognostic factors in primary resected early-stage non-small cell lung cancer. Virchows Arch. 2017; 470: 323-30.

13. Badowskakozakiewicz A, Sobol M, Patera J. Expression of Hypoxia-Inducible Factor 1a in Invasive Breast Cancer with Metastasis to Lymph Nodes: Correlation with Steroid Receptors, HER2 and EPO-R. Adv Clin Exp Med. 2016; 25: 741-50.

14. Ma CP, Liu H, Yi-Feng CI, et al. ADAR1 promotes robust hypoxia signaling via distinct regulation of multiple HIF-1alpha-inhibiting factors. EMBO Rep. 2019: 20: undefined.

15. Fu C, Tyagi R, Chin AC, et al. Inositol polyphosphate multikinase inhibits angiogenesis via Inositol Pentakisphosphate-Induced HIF-1a degradation. Circ Res. 2018; 122: 457-72
16. Han T, Yan J, Chen $\mathrm{H}$, et al. HIF-1alpha contributes to tube malformation of human lymphatic endothelial cells by upregulating VEGFR-3. Int J Oncol. 2019; 54: 139-51.

17. Schito L, Rey S, Tafani M, et al. Hypoxia-inducible factor 1-dependent expression of platelet-derived growth factor B promotes lymphatic metastasis of hypoxic breast cancer cells. Proc Natl Acad Sci USA. 2012; 109: E2707-16.

18. Beishline K, Azizkhan-Clifford J. Sp1 and the 'hallmarks of cancer'. FEBS J. 2015; 282: 224-58.

19. Lu XF, Li EM, Du ZP, et al. Specificity protein 1 regulates fascin expression in esophageal squamous cell carcinoma as the result of the epidermal growth factor/extracellular signal-regulated kinase signaling pathway activation. Cell Mol Life Sci. 2010; 67: 3313-29.

20. Safe S, Abdelrahim M. Sp transcription factor family and its role in cancer. Eur J Cancer. 2005; 41: 2438-48.

21. hi H, Li Y, Feng G, et al. The oncoprotein HBXIP up-regulates FGF4 through activating transcriptional factor Sp1 to promote the migration of breast cancer cells. Biochem Biophys Res Commun. 2016; 471: 89-94.

22. Blick $\mathrm{C}$, Ramachandran A, Mccormick $\mathrm{R}$, et al. Identification of a hypoxia-regulated miRNA signature in bladder cancer and a role for miR-145 in hypoxia-dependent apoptosis. Br J Cancer. 2015; 113: 634-44.

23. Pawlus MR, Wang L, Hu CJ. STAT3 and HIF1 alpha cooperatively activate HIF1 target genes in MDA-MB-231 and RCC4 cells. Oncogene. 2014; 33: 1670-9.

24. Vaupel P. The role of hypoxia-induced factors in tumor progression. Oncologist. 2004; 9: 10-7.

25. Lin C, Song L, Liu A, et al. Overexpression of AKIP1 promotes angiogenesis and lymphangiogenesis in human esophageal squamous cell carcinoma. Oncogene. 2015; 34: 384-93.

26. Xiang J, Zang W, Che J, et al. Regulation network analysis in the esophageal squamous cell carcinoma. Eur Rev Med PharmacolSci. 2012; 16: 2051-6.

27. Yu C, Zhou YX, Xiong P, et al. Functional haplotypes in the promoter of matrix metalloproteinase-2 predict risk of the occurrence and metastasis of esophageal cancer. Cancer Res. 2004; 64: 7622-8.

28. Shi M, Chen D, Yang D, et al. CCL21-CCR7 promotes the lymph node metastasis of esophageal squamous cell carcinoma by up-regulating MUC1. J Exp Clin Cancer Res. 2015; 34: 149.

29. Zhou C, Ye L, Jiang C, et al. Long noncoding RNA HOTAIR, a hypoxia-inducible factor-1a activated driver of malignancy, enhances hypoxic cancer cell proliferation, migration, and invasion in non-small cell lung cancer. Tumour Biol. 2015; 36: 9179-88.

30. Ji, RC. Hypoxia and lymphangiogenesis in tumor microenvironment and metastasis. Cancer Lett. 2014. 346: 6-16.

31. Paduch R. The role of lymphangiogenesis and angiogenesis in tumor metastasis. Cell Oncol (Dordr). 2016; 39: 397-410.

32. Ribeiro M, Teixeira SR, Azevedo MN, et al. Expression of hypoxia-induced factor-1 alpha in early-stage and in metastatic oral squamous cell carcinoma. Tumour Biol. 2017. 39: 175-80

33. Rankin EB, Giaccia AJ. Hypoxic control of metastasis. Science. 2016; 352:175-80.

34. Gong L, Zhang W, Zhou J, et al. Prognostic value of HIFs expression in head and neck cancer: a systematic review. PLoS One. 2013; 8: e75094.

35. Geis T, Popp R, Hu J, et al. HIF-2alpha attenuates lymphangiogenesis by up-regulating IGFBP1 in hepatocellular carcinoma. Biol Cell. 2015; 107: 175-88.

36. Guimaraes TA, Farias LC, Santos ES, et al. Metformin increases PDH and suppresses HIF-1alpha under hypoxic conditions and induces cell death in oral squamous cell carcinoma. Oncotarget, 2016; 7: 55057-68.

37. Munoz-Guerra MF, Fernandez-Contreras ME, Moreno $\mathrm{AL}$, et al. Polymorphisms in the hypoxia inducible factor 1-alpha and the impact on the prognosis of early stages of oral cancer. Ann Surg Oncol. 2009; 16: 2351-8.

38. Wang Y, Hua Q. Clinical significance of HIF-1 alpha,VEGF and VEGF-C expression in papillary thyroid carcinoma. Lin Chung Er Bi Yan Hou Tou Jing Wai Ke Za Zhi. 2007; 21: 204-6.

39. Zhang J, Zhu ZG, Ji J, et al. Transcription factor Sp1 expression in gastric cancer and its relationship to long-term prognosis. World J Gastroenterol. 2005; 11: 2213-7.

40. Qi F, Liu X, Wu H, et al. Long noncoding AGAP2-AS1 is activated by SP1 and promotes cell proliferation and invasion in gastric cancer. J HematolOncol. 2017; 10: 48 .

41. $\mathrm{Hu} \mathrm{J}, \mathrm{Hu} \mathrm{H}, \mathrm{Han} \mathrm{JJ}$, et al. Simultaneous high expression of PLD1 and Sp1 predicts a poor prognosis for pancreatic ductal adenocarcinoma patients. Oncotarget. 2016; 7: 78557-65.

42. Takami Y, Russell MB, Gao C, et al. Sp1 regulates osteopontin expression in SW480 human colon adenocarcinoma cells. 2007; 142: 163-9.

43. Tong Y, Tan Y, Zhou C, et al. Pituitary tumor transforming gene interacts with Sp1 to modulate G1/S cell phase transition. Oncogene. 2007; 26:5596-605.

44. Xu J, Zhou JY, Wei WZ, et al. Sp1-Mediated TRAIL Induction in Chemosensitization. Cancer Res. 2008; 68: 6718-26.

45. Hsu TI, Wang MC, Chen SY, et al. Sp1 expression regulates lung tumor progression. Oncogene. 2012; 31:3973-88

46. Li L, Gao P, Li Y, et al. JMJD2A-dependent silencing of Sp1 in advanced breast cancer promotes metastasis by downregulation of DIRAS3. Breast Cancer Res Treat. 2016; 156: 207-8.

47. $\mathrm{Wu} \mathrm{W}, \mathrm{Hu} \mathrm{Q}, \mathrm{Nie} \mathrm{E}$, et al. Hypoxia induces $\mathrm{H} 19$ expression through direct and indirect Hif-1a activity, promoting oncogenic effects in glioblastoma. Sci Rep. 2017; 7: 45029 
48. Woo SK, Kwon MS, Geng Z, et al. Sequential activation of hypoxia-inducible factor 1 and specificity protein 1 is required for hypoxia-induced transcriptional stimulation of Abcc8. J Cereb Blood Flow Metab. 2012; 32: 525-36.

49. Ishii $\mathrm{H}$, Chikamatsu K, Sakakura K, et al. Primary tumor induces sentinel lymph node lymphangiogenesis in oral squamous cell carcinoma. Oral Oncol. 2010; 46: 373-8.

50. Wakisaka N, Hasegawa Y, Yoshimoto S, et al. Primary Tumor-Secreted Lymphangiogenic Factors Induce Pre-Metastatic Lymphvascular Niche Formation at Sentinel Lymph Nodes in Oral Squamous Cell Carcinoma. PLoS One. 2015; 10: e0144056.

51. Fu X, Zhai S, Yuan J. Interleukin-6 (IL-6) triggers the malignancy of hemangioma cells via activation of HIF-1alpha/VEGFA signals. Eur J Pharmacol. 2018; 841: 82-9.

52. Cheng HT, Hung WC. Inhibition of lymphangiogenic factor VEGF-C expression and production by the histone deacetylase inhibitor suberoylanilide hydroxamic acid in breast cancer cells. Oncol Rep. 2013; 29: $1238-44$. 